\title{
Viburnum Sargentii Koehne Fruit Extract As Corrosion Inhibitor For Mild Steel In Acidic Solution
}

\author{
Xiumei Wang ${ }^{*}$, Yichong Wang, Qing Wang, Ye Wan, Xiaoqi Huang, Chen Jing \\ School of Materials Science and Engineering, Shenyang Jianzhu University, Shenyang, Liaoning, \\ China 110168 \\ *E-mail: xmwang@alum.imr.ac.cn
}

doi: $10.20964 / 2018.06 .36$

Received: 5 January 2018 / Accepted: 31 March 2018 / Published: 10 May 2018

\begin{abstract}
The inhibition effect of Viburnum sargentii Koehne fruit extract (JMG) on mild steel corrosion in $1 \mathrm{M}$ $\mathrm{HCl}$ solution was studied by weight loss measurement, electrochemical impedance spectroscopy (EIS) and potentiodynamic polarization curves. The results show that the inhibition effect has a relationship with the dosage of JMG. The inhibition efficiency increases as the concentration of JMG increases and exhibits the opposite trend with an increment in the temperature. The maximum inhibition efficiency reaches $93.8 \%$ with the addition of $2.0 \% \mathrm{JMG}$ at $303 \mathrm{~K}$ (weight loss results). JMG inhibits the anodic and cathodic processes simultaneously, and $E_{\text {corr }}$ does not change with the increasing concentration, indicating JMG exhibits mixed-type inhibition behavior. The adsorption process of JMG obeys the Langmuir isotherm model, which is an exothermic physisorption accompanied by a decrease in entropy. The activation energy determines the corrosion rate of mild steel in $1 \mathrm{M} \mathrm{HCl}$ solution.
\end{abstract}

Keywords: Corrosion Inhibitor; Extract of Viburnum sargentii Koehne Fruit; Mild Steel; Acidic Medium; Electrochemical Method

\section{$\underline{\text { FULL TEXT }}$}

(C) 2018 The Authors. Published by ESG (www.electrochemsci.org). This article is an open access article distributed under the terms and conditions of the Creative Commons Attribution license (http://creativecommons.org/licenses/by/4.0/). 